\title{
Community Detection in Complex Network via BGLL Algorithm
}

\author{
Pooja Chaturvedi \\ M.Tech(CSE) \\ ASET,Amity University \\ Lucknow
}

\author{
Mousumi Dhara \\ Research Scholar \\ IT-BHU \\ Varanasi
}

\author{
Deepak Arora, PhD. \\ Assistant Professor \\ ASET,Amity University \\ Lucknow
}

\begin{abstract}
A large number of networks in nature, society and technology are defined by a mesoscopic level of organization, in which groups of nodes form tightly connected units, called communities, that are sparsely inter-linked to each other .Identifying this community structure is one of the most important problems in understanding of functions and structures of real world complex systems, which is still a challenging task. Various methods proposed so far are not efficient and accurate for large networks which comprise of millions of nodes because of their high computational cost.
\end{abstract}

In this manuscript we will provide the implementation and behavioral analysis of BGLL algorithm for determining the structure of complex networks. This method is a variant of hierarchical agglomerative clustering approach, which finds the communities which are nested within one another. This method emphasizes on the idea of building the communities by combining the initial partition into super networks by repeatedly optimizing the modularity.

In this work we will implement the BGLL Algorithm on various large networks which exhibit the community structure .We will also determine the optimal modularity at every pass and determine the hierarchical structure of large complex systems that comprise of millions of nodes. We will also provide a brief comparison of BGLL algorithm with some methods.

\section{General Terms}

Graph Theory, Data Mining.

\section{Keywords}

Complex Networks, Community Structure.

\section{INTRODUCTION}

Representation of various real world systems as graphs can give deep insight to the understanding the structure and functions of complex networks. Identification of Community structure has gained a lot of attention among the researchers, along with the other properties of these networks such as small world effect, scale free, power-law degree distribution. A network is said to possess community structure if the edge density within the community is sufficiently larger than between the communities.

Clustering is considered as an important task in the study of complex network's properties. Merriam Webster 2008 defines clustering as a "statistical classification technique for determining whether the elements of a cluster fall into different groups by ensuring quantitative comparison of various features. A number of clustering approaches are available in literature. Broadly these approaches can be divided in two groups: (i) Hierarchical Clustering approach and (ii) Partition Clustering approach. Hierarchical clustering algorithms recursively find interconnected clusters in either Agglomerative manner(Bottom-up) or Divisive manner(TopDown) Berkhin (2009) found six more approaches in addition to above two groups :1.Grid-Based Methods 2.Methods based on co-occurrence of categorical data 3.Constraint Based Clustering 4. Clustering algorithms used in machine learning 5.Scalable Clustering algorithms 6.Algorithms for High Dimensional Data. Clustering founds its application in various fields including information retrieval, image segmentation, character recognition, VLSI design, computer graphics and gene analysis. Graph Clustering applications include: monitoring computer networks for administration purposes ,visualizing knowledge bases to support human understanding, clustering metric data etc.

\section{RELATED WORK}

Newman and Girvan's work in 2001 suggested that complex systems posses the property of community structure. Identification and detection of community structure was originated as the extension of the problem of graph partitioning. Graph partitioning problem comprised of the division of graph nodes into predetermined number of partitions such that the number of edges between the groups is as few as possible. The number of edges between the groups is called as cut size. Kernighan $\mathrm{Li}$ approach is the most popular method for this problem. Spectral bisection is another approach for this problem, which is based on the Laplacian matrix. Another approach for graph partitioning is based on the min cut max flow method proposed by Ford and Fulkerson. Min cut and max flow approach is used in determining communities in the web networks by Flake et.al. Graph can be partitioned into groups by measuring the conductance which affine to the cut size of the graph.

Conductance is computed as:

$\varphi(\mathrm{C})=(\mathrm{c}(\mathrm{C}, \mathrm{g} / \mathrm{C})) /\left(\min \left(\mathrm{k} \_\mathrm{C}, \mathrm{k} \_(\mathrm{g} / \mathrm{C})\right)\right)$

Where $\mathrm{c}(\mathrm{C}, \mathrm{g} \mid \mathrm{C})$ is the cut size of $\mathrm{C}$, and $\mathrm{k} \_(\mathrm{g} \mid \mathrm{C})$ are the total degrees of $\mathrm{C}$ and of the rest of the graph $\mathrm{glC}$, respectively.

Graph partitioning approaches are not good for community detection, as these methods require the prior information about the number of subparts into which the graph is to be divided. The traditional methods of community detection were hierarchical clustering. Hierarchical clustering methods may be of two types either agglomerative or divisive. 
Newman proposed a method of community detection which was based on the idea of betweenness measure. The betweenness was calculated for each pair of edges and edges with the maximum betweenness removed. This process was repeated until no edge remains. Clauset, Newman and Moore proposed the fast modularity approach for community detection which basically finds the best pair of communities to merge and what will be the criteria to stop merging the communities. In this method three data structures were maintained:

1. A sparse matrix Q containing the modularity increment for every pair of communities having a common edge.

2 A max heap consists of the maximum element of each row of Q.

\section{An ordinary vector array a.}

The method starts by calculating the initial values of matrix $\mathrm{Q}$ and with the help of following formulas:

$$
\begin{gathered}
\Delta Q_{\mathrm{ij}}=\left\{\begin{array}{c}
\frac{1}{2 \mathrm{~m}}-\frac{\mathrm{k}_{\mathrm{i}} \mathrm{k}_{\mathrm{j}}}{(2 \mathrm{~m})^{2}}, \quad \text { if } i \text { and } j \text { are connected } \\
0, \text { otherwise }
\end{array}\right. \\
\mathrm{a}_{\mathrm{i}}=\frac{\mathrm{k}_{\mathrm{i}}}{2 \mathrm{~m}}
\end{gathered}
$$

Then we select the largest element from each row of the matrix and merge the related communities. This process is repeated until there is one community remains. The running time of this method is $\mathrm{O}(\operatorname{mdlog} n)$. The extension of CNM algorithm was proposed by $\mathrm{Xu} \mathrm{Liu}$ in their seminal paper, where they used an additional parameter head-size with the heap data structure. The head size controlled the randomness in the search path and search strategies. Pons and Latapy used the idea of random walks across the network for community detection. Instead of using modularity as similarity measure this method used the node similarity measure based on short walks. This method was also an instance of hierarchical aggregation. Walk trap method has the complexity of $\mathrm{O}(\mathrm{mn})^{2}$ which could be reduced to $\mathrm{O}\left(\mathrm{n}^{4}\right)$.

Markov clustering algorithm was implemented as a simulation of flow through the network. The underlying idea of this method is that the random walker will spend more time inside the same community. The Markov clustering algorithm has the complexityO $\left(\mathrm{n}^{3}\right)$. Karsten and Nitesh V.Chawla proposed a method based on random walks. It assumed that a random walker with predetermined number of steps is likely walk inside a single community. The idea of this method was to compute many random short walks and keep tracks of the visited nodes in a single walk.

\section{BGLL ALGORITHM}

\section{Overview of the method}

This community detection algorithm is mainly known as Louvain Method, named after its co-authors location. This method gained a lot of attention among the researchers because of its computational speed and accuracy of the communities detected. The idea of this method was given by Etienne Lefebvre in his Master's Thesis. This method was first improvised and studied by Blondel et al in their paper
"Fast Unfolding of Communities". They concluded in their study that this method was fast in determining the community structure for large networks. It efficiency was also tested on a large data set of Belgian Phone network and on adhocnetworks. It is a simple, efficient and fast method which has been tested on large networks consisting of millions of nodes. This method is considered as one of the most popular methods for community detection. Louvain method is considered as better method than other methods because it computes high modularity partitions and hierarchies of large networks in quick time. This method is more important because of its independence from the resolution limit problem, which was the major concern for all the other methods.

BGLL algorithm consists of two phases - In the first step each node belongs to its own cluster. The clusters are formed more specifically by finding the modularity change by moving a node into the group of neighborhood node. A node is placed into that group for which the modularity gain is positive and maximum. This process is applied for all nodes until there is no improvement possible in the value of modularity. In this modularity gain is computed by:

$$
\Delta \mathrm{Q}=\left[\frac{\sum_{\text {in }}+2 \mathrm{k}_{\mathrm{i}, \mathrm{in}}}{2 \mathrm{~m}}-\left(\frac{\sum_{\text {tot }}+\mathrm{k}_{\mathrm{i}}}{2 \mathrm{~m}}\right)^{2}\right]-\left[\frac{\sum \mathrm{in}}{2 \mathrm{~m}}-\left(\frac{\sum \mathrm{tot}}{2 \mathrm{~m}}\right)^{2}-\left(\frac{\mathrm{ki}_{\mathrm{i}}}{2 \mathrm{~m}}\right)^{2}\right]
$$

Where $\sum$ in is the sum of weights of the link inside $C, \sum$ tot $s$ the sum of the weights of the links incident to nodes in $\mathrm{C}, \mathrm{k}_{\mathrm{i}}$ is the sum of the weights incident to node $C, k_{i, \text { in }}$ is the sum of the weights of the links from node $\mathrm{i}$ to node in $\mathrm{C}, \mathrm{m}$ is the sum of the weights of all the links in the network.

The second phase comprise of building a new network by considering the communities found in first phase as nodes. The weight of the link between the two nodes is given by the sum of weights of the links in the communities. The two phases combined constitute a pass. After each the number of meta-communities decreases every time. The passes are repeated until no improvement can be achieved and the maximum modularity is achieved. The hierarchy of the structure is determined by the number of passes. The advantage of this method is that the calculation of modularity gain is simple in this method. The algorithm provides high modularity partition and hierarchical structure can be visualized at different resolution. Thus this method eliminates the resolution limit problem of earlier methods.

The algorithm consists of three main functions.

1. Convert: This function takes the graph in .txt format describing the nodes as a pair of source and destination nodes, as input and converts the graph in binary format.

2. Community: This is the heart of the algorithm, where the communities are computed according to the two phases as described above.

3. Hierarchy: This function describes the hierarchical structure of the networks in terms of number of levels and number of nodes at each level.

It is also possible to find the belongingness of nodes by using the function node2_comm_level at any level.

\section{Experimental Results 4.1 Tests on Real Networks}

We have tested the algorithm on these real networks. Table 1 provides the brief description of the networks. 
Table 2 represents the maximum modularity achieved and the number of communities detected by applying the algorithm on various real world networks.

\subsection{Hierarchical Organization of networks}

Table 3 represents the hierarchical structure of the networks as obtained after the application of the algorithm. It shows the number of nodes decrease in subsequent passes, indicating that nodes are combined to form a super-network of the nodes found in earlier pass. The process stops when there is no change in modularity i.e. when the optimum modularity is achieved.

\subsection{Size distribution with modularity change}

Figure 1 represents the size distribution of the nodes with the modularity class.

\subsection{Modularity Change in various passes}

Figure 2 represents the modularity change in the respective pass. Horizontal axis represents the number of pass and vertical axis represents the modularity change.

\subsection{Community Structure}

Figure 3 provides the visualization of the communities detected by the algorithm. We have used Gephi software for the visualization of communities.

\subsection{Hierarchical level of networks}

Figure 4 represents the hierarchical level in the form of drastic change in the number of nodes after few passes. The hierarchical level obtained provides the facility to have a deep insight to the network structure at multiple resolution.

\section{CONCLUSION AND DISCUSSION}

Graph partitioning approaches are based on the idea of maximum flow and minimum cut. It also imposes the constraint that clusters should be of equal size. This problem is known as minimal bisection problem. Studies have showed that finding the partitions of all equal size is a NP-hard problem. Kerninghan $\mathrm{Li}$ algorithm is the most popular method used for graph partitioning. It finds the random partition of predefined size. The subsets having equal number are swapped in the groups. After the swapping the group with the maximum modularity is selected. This method performs in quick time if there are a limited number of swaps.

Hierarchical clustering approaches are very popular for graph clustering. They can be either agglomerative or divisive. The problem of these methods is that they don't discriminate the partitions so well. Also as they don't consider neighborhood, so there may be the possibility of considering a node with a single neighbor as the separate clusters. A main drawback of agglomerative hierarchical clustering method is that it does not scale well, i.e. it doesn't perform accurately for large networks. If points are scattered ins space then distance can be used as a dissimilarity measure and the method runs in $\mathrm{O}\left(\mathrm{n}^{2}\right)$ for single linkage, $\mathrm{O}\left(\mathrm{n}^{2} \log \mathrm{n}\right)$ for complete and average linkage schemes. For graph clustering, where distance is not specifically defined these methods may have larger complexity. Another approach for graph clustering is spectral clustering, which is based on the computation of first $\mathrm{k}$ Eigen vectors of a Laplacian Matrix, which becomes practically difficult for large graphs.

Newman's betweenness method is divisive clustering approach. It is based on the idea of calculating the betweenness between each pair of edges. Here betweenness is referred to as the shortest distance between the edges. It works by removing the edge with the maximum betweenness and continues until all the edges have been taken into consideration. This algorithm has a drawback of large computation time, as it take the time complexity of the order of $\mathrm{O}\left(\mathrm{m}^{2} \mathrm{n}\right)$ or $\mathrm{O}\left(\mathrm{n}^{3}\right)$ for a large sparse graph.

Newman defined a new parameter to define the quality measure of the partition, which is known as modularity. Newman also proposed a new approach of graph clustering which was based on modularity optimization.

Table 1: Description of the data sets on which BGLL algorithm have been tested

\begin{tabular}{|l|l|}
\hline Name & Description \\
\hline Dolphin & $\begin{array}{l}\text { Contains an undirected social network of frequent } \\
\text { associations of 64 dolphins. }\end{array}$ \\
\hline Football & Contains the network of American football games \\
\hline As-22july06 & $\begin{array}{l}\text { Contains the schematic snapshot of the structure of Internet } \\
\text { at the levels of Autonomous systems. }\end{array}$ \\
\hline Netscience & $\begin{array}{l}\text { Contains a co-authorship network of scientists working on } \\
\text { network theory and experiment. }\end{array}$ \\
\hline Polblogs & $\begin{array}{l}\text { Collection of political blogs and links during the time of } \\
\text { elections. }\end{array}$ \\
\hline Celegansneural & Contains the weighted directed neural network of C.Elegan. \\
\hline
\end{tabular}


Table 2.Table represents the optimal modularity achieved in each networks and the number of communities detected.

\begin{tabular}{|l|l|l|l|l|l|}
\hline Name & No. of Nodes & No. of Links & Weights & Modularity & Communities \\
\hline Dolphins & 64 & 160 & 320 & 0.524531 & 5 \\
\hline Football & 117 & 614 & 1228 & 0.605156 & 10 \\
\hline As-22july06 & 22965 & 48437 & 96874 & 0.660659 & 29 \\
\hline Netscience & 1463 & 2743 & 5486 & 0.959522 & 403 \\
\hline Polblogs & 1225 & 16717 & 33434 & 0.426983 & 277 \\
\hline Celegansneural & 298 & 2149 & 4298 & 0.393309 & 5 \\
\hline
\end{tabular}

Fig1: Size-distribution vs Modularity class graph of networks: a) Dolphins b) Football c) as-22july06 d) Net-science e) Polblog f) Celegans-neural network. These results have been obtained by setting the Resolution limit= 1.0 and Randomize value on. Edge weights are also considered.

\section{Results:}

Modularity: 0.520

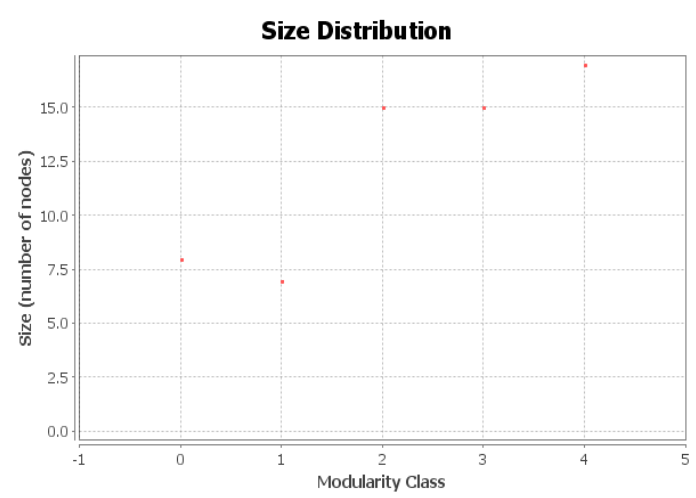

Results:

Modularity: 0.654
Number of Communities:

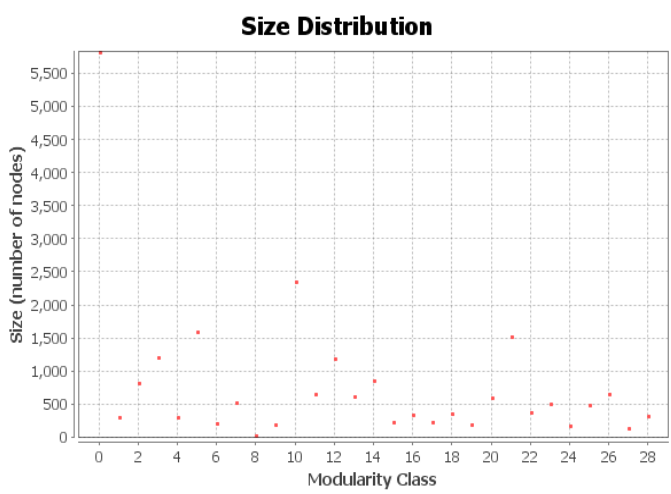

\section{Results:}

Modularity: 0.605
Number of Communities: 10

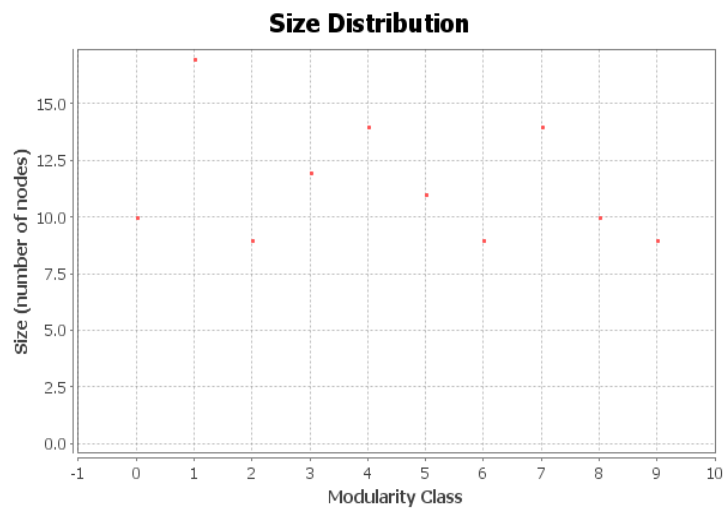

Results:

Modularity: 0.958
Number of Communities: 403

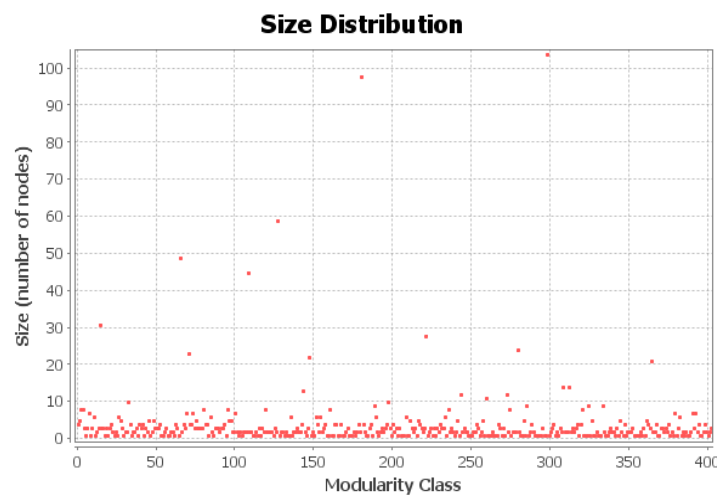


Results:

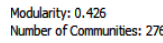

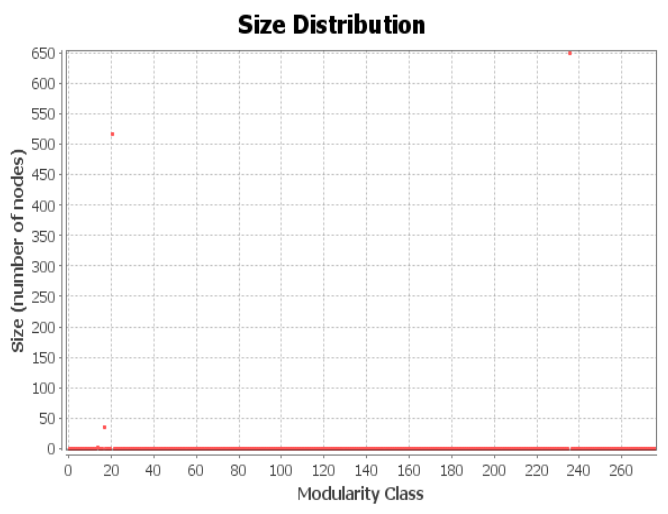

Results:

Modularity: 0.460
Number of Communities: 5

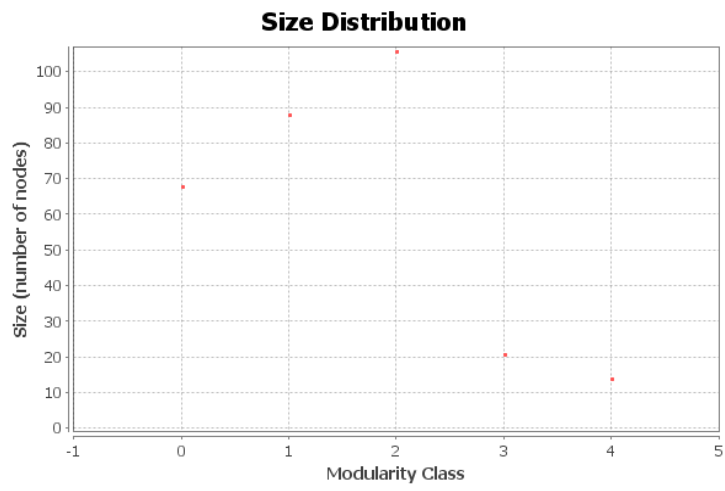

Fig 2: Community structure of real world networks---a) Dolphins b) Football c) Netscience d) Polblog e) Celegansneural.
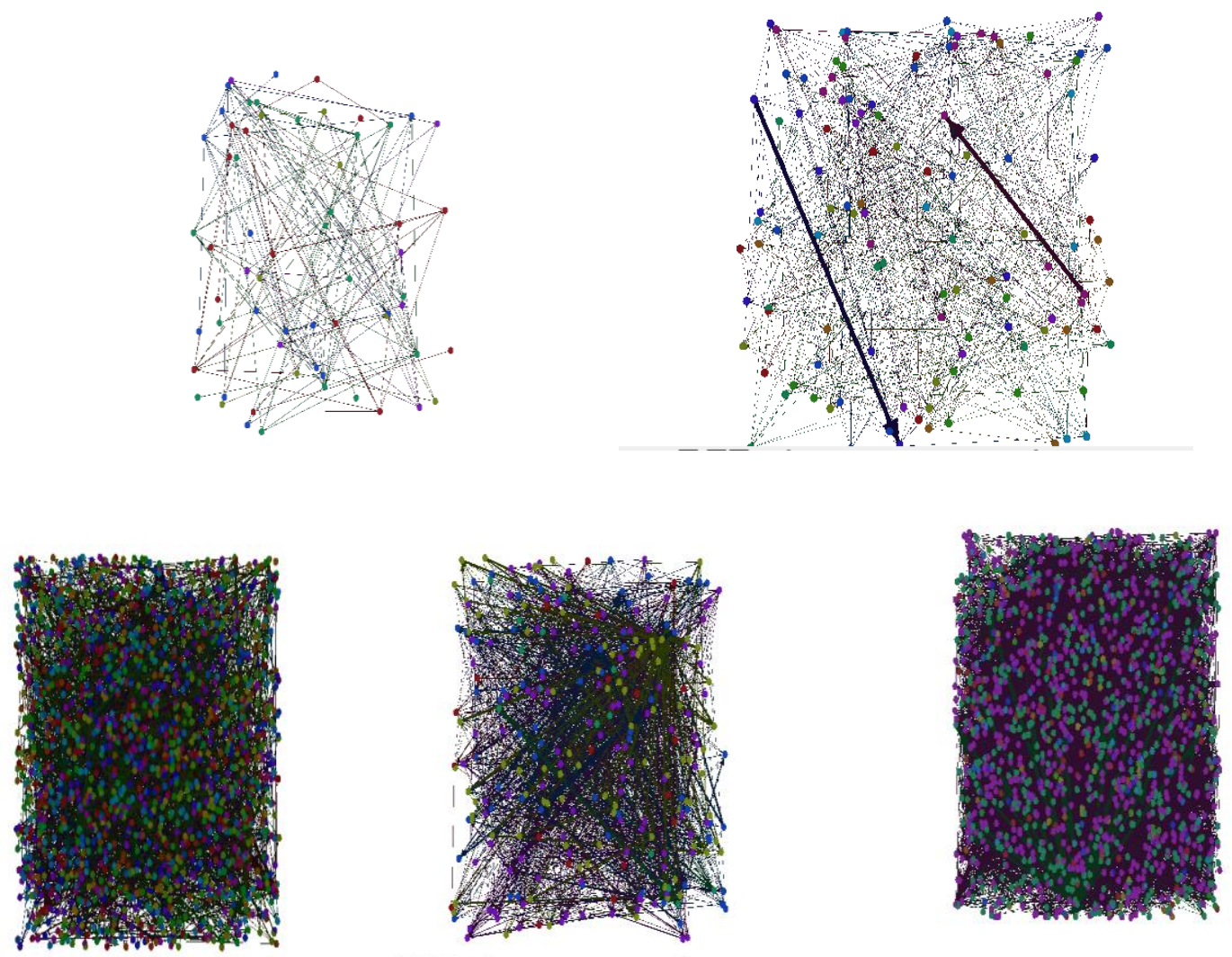
Table 3 : Hierarchical level of netwroks with shrinking nodes.From this table we may conclude that the number of passes is at most 5 .

\begin{tabular}{|c|c|}
\hline Name & Hierarchical level \\
\hline Dolphins & $\begin{array}{l}\text { No. of levels:3 } \\
\text { Level0:64 nodes } \\
\text { Level1:10 nodes } \\
\text { Level2:6 nodes }\end{array}$ \\
\hline Football & $\begin{array}{l}\text { No. of levels:3 } \\
\text { Level0:117 nodes } \\
\text { Level1:15 nodes } \\
\text { Level2 }: 11 \text { nodes }\end{array}$ \\
\hline As-22july06 & $\begin{array}{l}\text { No. of levels: } 4 \\
\text { Level0:22965 nodes } \\
\text { Level1:2839nodes } \\
\text { Level2:260nodes } \\
\text { Level3:61nodes }\end{array}$ \\
\hline Netscience & $\begin{array}{l}\text { No. of levels: } 4 \\
\text { Level0:1463 nodes } \\
\text { Level1:375 nodes } \\
\text { Level2:289 nodes } \\
\text { Level3:279 nodes }\end{array}$ \\
\hline Polblog & $\begin{array}{l}\text { No. of levels: } 4 \\
\text { Level0:1225nodes } \\
\text { Level1:27 nodes } \\
\text { Level2:11 nodes } \\
\text { Level3:10 nodes }\end{array}$ \\
\hline Celegansneural & $\begin{array}{l}\text { No. of levels:3 } \\
\text { Level0:298 nodes } \\
\text { Level1:13 nodes } \\
\text { Level2:5 nodes }\end{array}$ \\
\hline
\end{tabular}


a) Dolphins Network
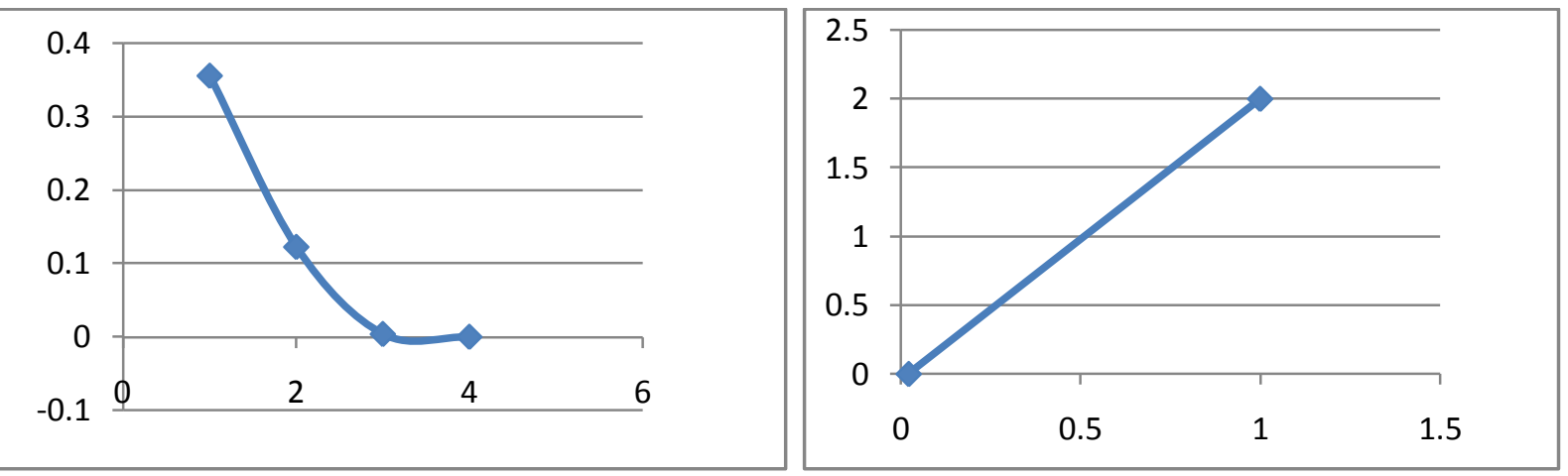

Pass 1

Pass 2

b) Football Network

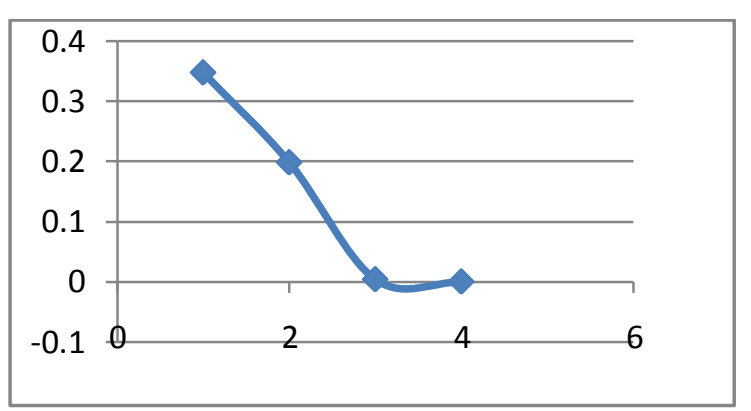

Pass 1

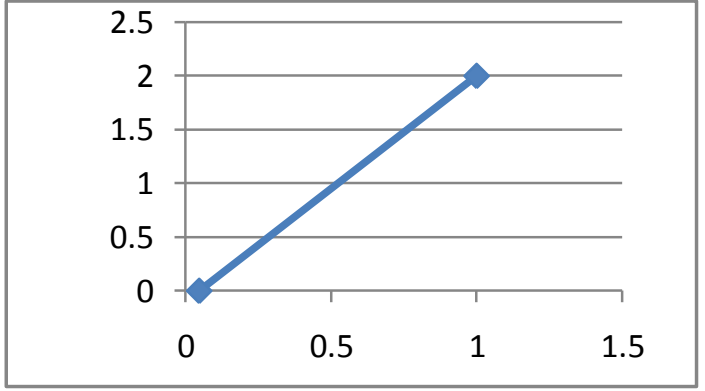

Pass 2

\section{c) Netscience}

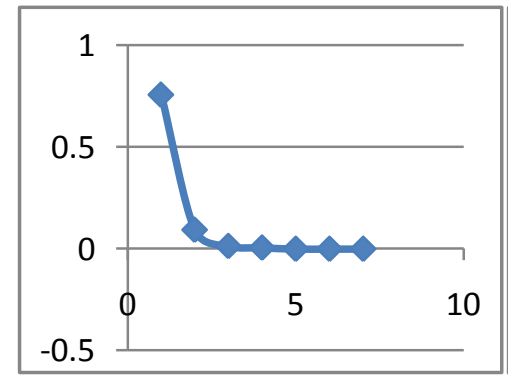

Pass 1

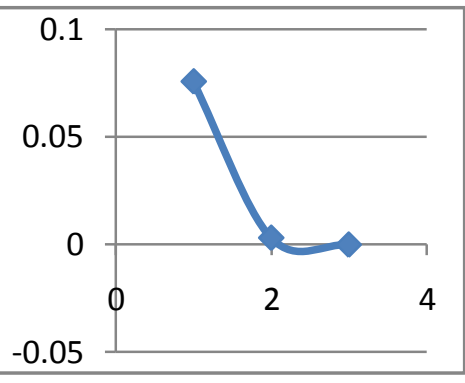

Pass 2

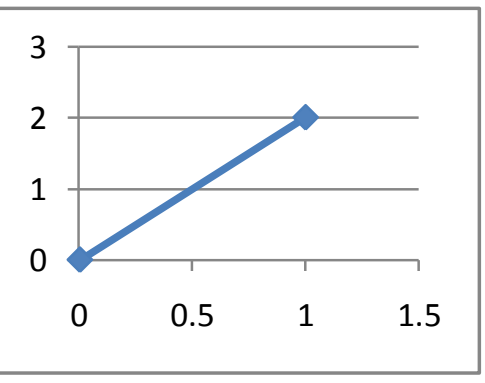

Pass 3

d) Polblog

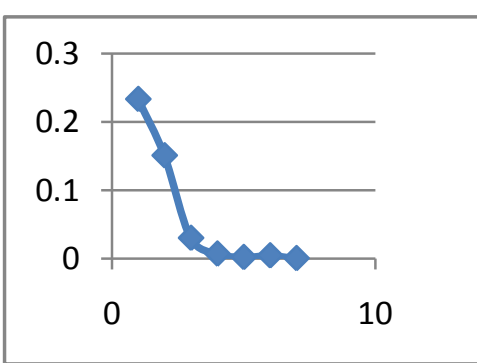

Pass 1
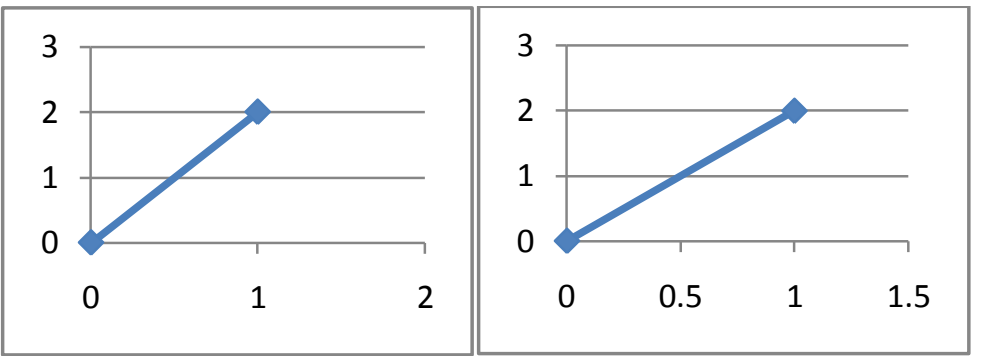
e) Celegans Neural

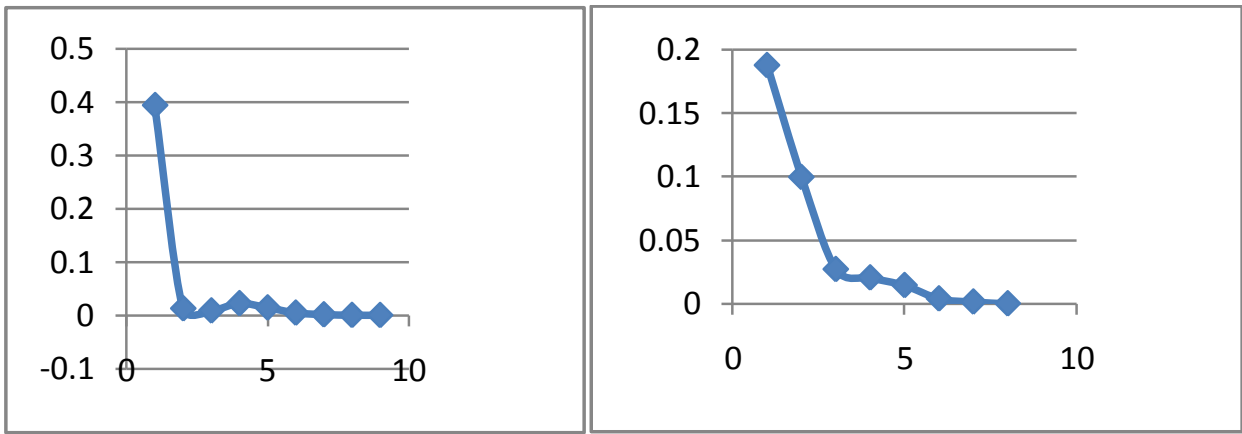

Pass 1

Pass 2

\section{f) As-22july06}

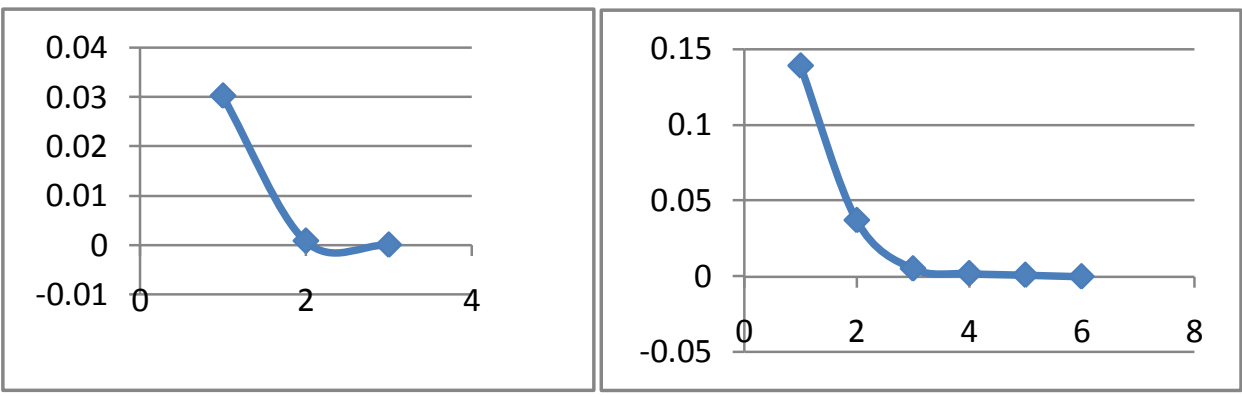

Pass 1

Pass 2

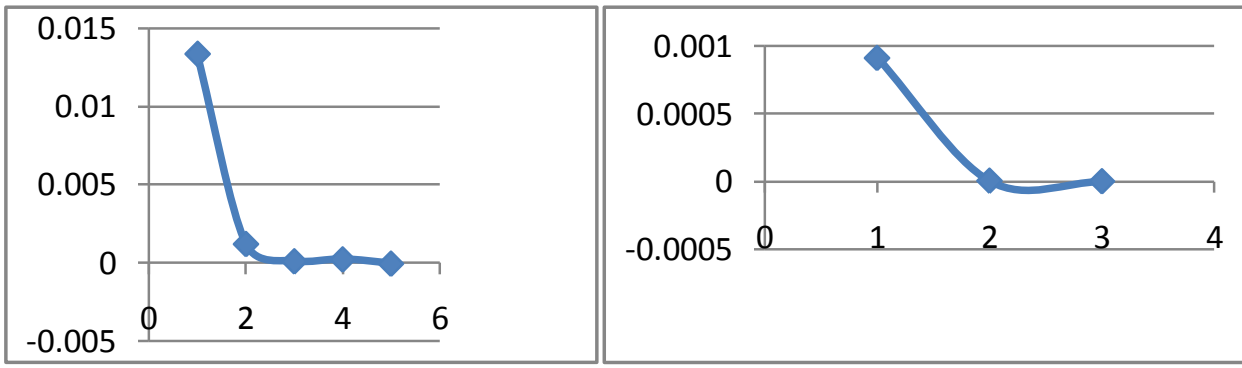

Pass 3

Pass 4

Figure 2 Modularity Change in various passes in networks 


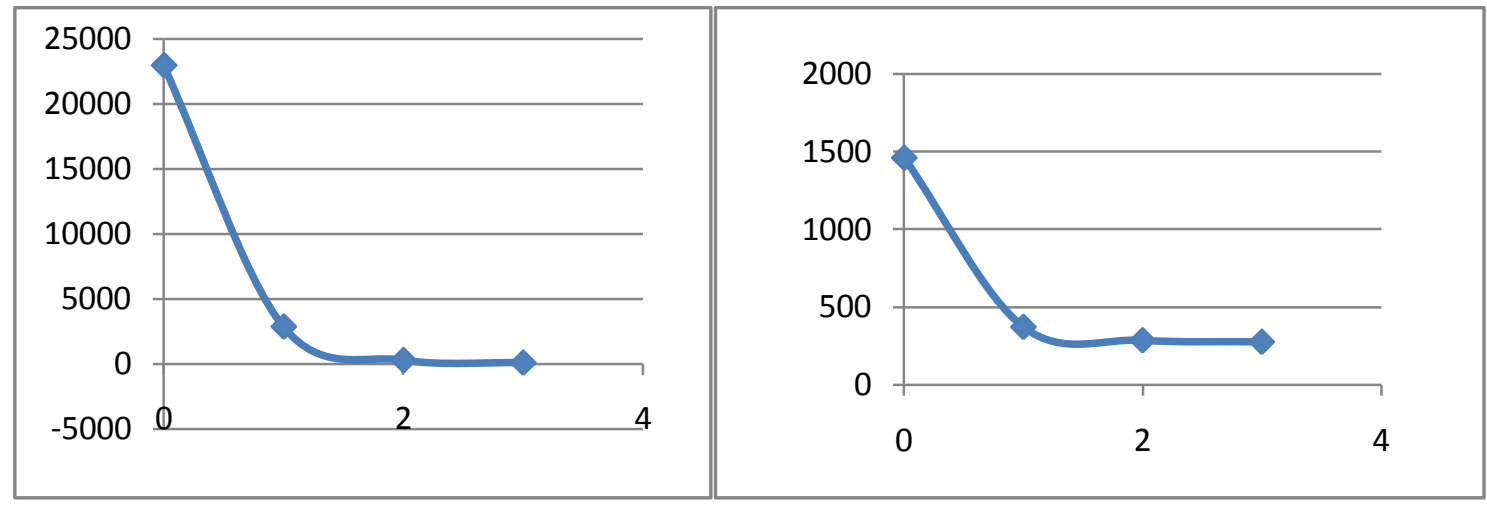

(a)

(b)

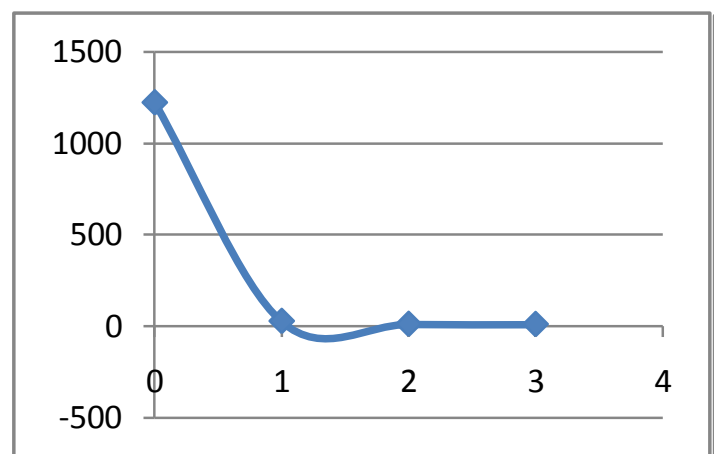

(c)

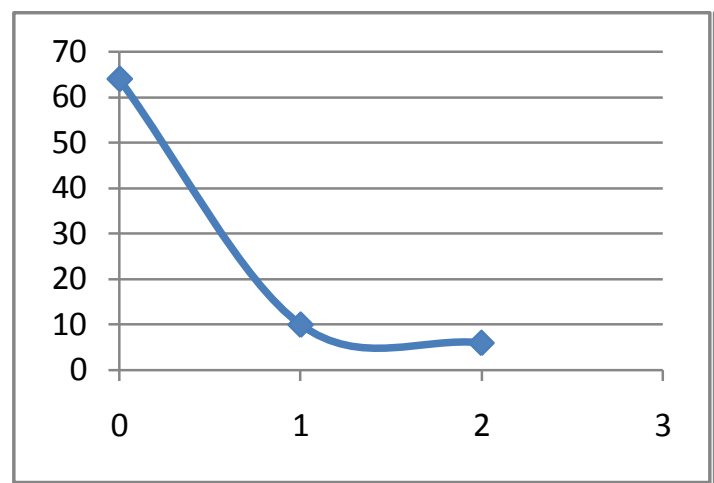

(e)

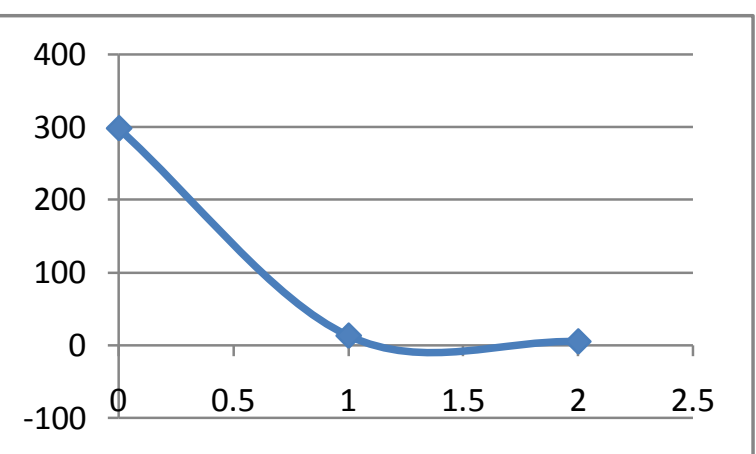

(d)

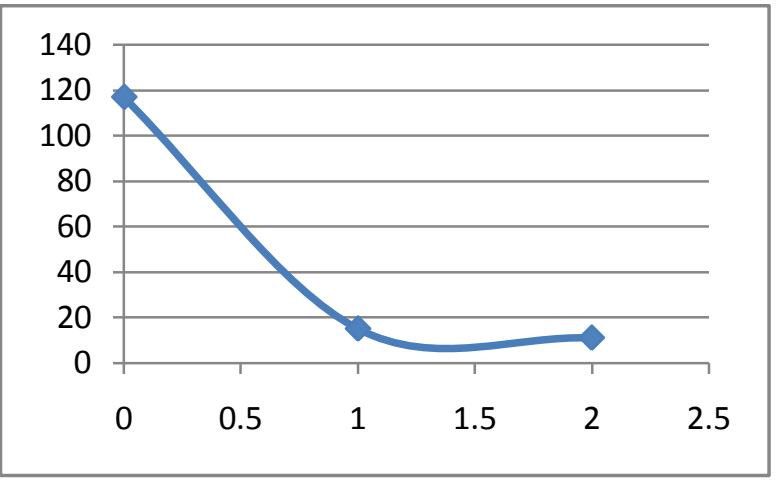

(f)

Figure 3: Hierarchical level of networks with shrinking nodes ---a) as-22july06 b) netscience c) polblogs d) celegansneural e) dolphins f) football. In this graph horizontal axis represents the number of pass and vertical axis represents the number of nodes.

In this method a matrix describing the modularity change between two nodes $\mathrm{i}$ and $\mathrm{j}$ was maintained. The two communities are merged if they have the highest modularity change. This method was now able to perform clustering for large network, But later Clauset et.al. work proposed that the calculation of modularity change required many useless operations in between, due to the sparsity of adjacency matrix, hence increasing the time complexity. Clauset introduced max-heap to maintain these updates. The greedy modularity optimization ignores the communities of small size and it quickly found large clusters, which account to poor values of modularity maximum. We remark that in BGLL algorithm modularity is always computed from the initial graph topology, operating on supergraphs enables one to consider the variations of modularity for partitions of the original graph after merging and/or splitting of groups of vertices. Therefore, at some iteration, modularity cannot increase any more, and the algorithm stops. The technique is more limited by storage demands than by computational time. The latter grows like $\mathrm{O}(\mathrm{m})$, so the algorithm is extremely fast and graphs with up to $10^{9}$ edges can be analyzed in a reasonable time on current computational resources. The modularity maxima found by 
the method are better than those found with the greedy techniques by Clauset et al. and Wakita and Tsurumi.

One thing to be note about our method is that the outcome depends on the order in which the nodes are considered. Although the order doesn't affect the modularity obtained in each pass, but it may affect the computation time. So in order to enhance the computation time, some threshold of the modularity is to be considered as stopping criteria.

The hierarchical level of networks is determined by the number of passes. From the results, we may conclude that the number of passes is generally a small number. The experimental result shows that the algorithm is extremely fast, as the complexity of this method is linear for typical and sparse data-sets.

\section{FUTURE WORK}

In this work we have tested the efficiency of BGLL on various large real networks. The limitation of this method was the storage of networks in the main memory than the computation time. We have also found that our algorithm performs well than earlier methods in terms of modularity optimization and computation time. The speed of this algorithm can be further increased by stopping the first phase when the certain threshold of the modularity is achieved. We can also increase the computation speed by removing the nodes of degree 1 from the network initially and adding them further after the community computation.

BGLL algorithm only detects the disjoint communities, whereas in real networks nodes may belong to more than one community. In the future this algorithm may be used for calculating the initial partition of network and further constraints may be applied to identify and analyze the overlapping communities. Also our method doesn't detect the hubs and outliers in the networks. Hubs are defined as the nodes which provide the link between the two communities, whereas outliers are the nodes that are marginally connected to the communities. So the method can be further refined to detect the hub and outliers.

\section{ACKNOWLEDGEMENT}

We are very thankful to Mr. Aseem Chauhan, Additional President, Amity University, Lucknow, Maj. Gen. K.K. Ohri, AVSM (Retd.), Director General, Amity University, Lucknow, India, for providing excellent computation facilities in the University campus. We also pay our regards to Prof. S.T.H. Abidi, Director and Brig. U.K. Chopra,Deputy Director, Amity School of Engineering, Amity University, Lucknow for giving their moral support and help to carry out this research work. We are very thankful to Blondel et. Al and Newman et al. for their work as it provides us the good understanding of the community detection concept.

\section{REFERENCES}

[1] L. Euler, Solutio problematis ad geometriam situs pertinentis, Commentarii Academiae Petropolitanae 8 (1736) 128-140.

[2] R. Albert, A.-L. Barabási, Statistical mechanics of complex networks, Rev. Mod. Phys. 74 (1) (2002) 47-97.

[3] M.E.J. Newman, The structure and function of complex networks, SIAM Rev. 45 (2) (2003) 167-256.University Press, Cambridge, UK, 2008.

[4] P. Erdös, A. Rényi, On random graphs. I., Publ. Math. Debrecen 6 (1959) 290297.
[5] S. Fortunato, C. Castellano, Community structure in graphs, in: R.A. Meyers (Ed.), Encyclopedia of Complexity and Systems Science, vol. 1, Springer,Berlin, Germany, 2009, eprint arXiv:0712.2716.

[6] G.W. Flake, S. Lawrence, C. Lee Giles, F.M. Coetzee, Self-organization and identification of web communities, IEEE Computer 35 (2002) 6671.

[7] R.S. Weiss, E. Jacobson, A method for the analysis of the structure of complex organizations, Am. Sociol. Rev. 20 (1955) 661668

[8] S.A. Rice, The identification of blocs in small political bodies, Am. Polit. Sci. Rev. 21 (1927) 619627.

[9] G.C. Homans, The Human Groups, Harcourt, Brace \& Co, New York, 1950

[10] M.E.J. Newman, M. Girvan, Finding and evaluating community structure in networks, Phys. Rev. E 69 (2) (2004) 026113

[11] E.A. Leicht, M.E.J. Newman, Community structure in directed networks, Phys. Rev. Lett. 100 (11) (2008) 118703 .

[12] M. Latapy, P. Pons, Lect. Notes Comput. Sci. 3733 (2005) 284293.

[13] B.H. Good, Y. de Montjoye, A. Clauset, The performance of modularity maximization in practical contexts, eprint arXiv:0910.0165.

[14] M.E.J. Newman, Finding community structure in networks using the eigenvectors of matrices, Phys. Rev. E 74 (3) (2006) 036104.

[15] B.W. Kernighan, S. Lin, An efficient heuristic procedure for partitioning graphs, Bell Syst. Tech. J. 49 (1970) 291307

[16] G.W. Flake, S. Lawrence, C.L. Giles, Efficient identification of web communities, in: Sixth ACM SIGKDD International Conference on Knowledge Discovery and Data Mining, ACM Press, Boston, USA, 2000, pp. 150160.

[17] M.E.J. Newman, A measure of betweenness centrality based on random walks, Soc. Netw. 27 (2005) 3954.

[18] M.E.J. Newman, Analysis of weighted networks, Phys. Rev. E 70 (5) (2004) 056131.

[19] M.E.J. Newman, Fast algorithm for detecting community structure in networks, Phys. Rev. E 69 (6) (2004) 066133 .

[20] A. Clauset, M.E.J. Newman, C. Moore, Finding community structure in very large networks, Phys. Rev. E 70 (6) (2004) 066111.

[21] K. Wakita, T. Tsurumi, Finding community structure in mega-scale social networks, eprint arXiv:cs/0702048.

[22] V.D. Blondel, J.-L. Guillaume, R. Lambiotte, E. Lefebvre, Fast unfolding of communities in large networks, J. Stat. Mech. P10008 (2008).

[23] S. White, P. Smyth, A spectral clustering approach to finding communities in graphs, in: Proceedings of SIAM International Conference on Data Mining, 2005, pp. 7684 . 
[24] M.E.J. Newman, From the cover: Modularity and community structure in networks, Proc. Natl. Acad. Sci. USA 103 (2006) 85778582.

[25] M.E.J. Newman, The structure of scientific collaboration networks, Proc. Natl. Acad. Sci. USA 98 (2) (2001) 404409.

[26] J. G. White, E. Southgate, J. N. Thompson, and S. Brenner, "The structure of the nervous system of the nematode C. Elegans", Phil. Trans. R. Soc.London 314, $1-340$ (1986)

[27] D. J. Watts and S. H. Strogatz, "Collective dynamics of "small-world' networks", Nature 393, 440-442 (1998).

[28] D. Lusseau, K. Schneider, O. J. Boisseau, P. Haase, E. Slooten, and S. M. Dawson, The bottlenose dolphin community of Doubtful Sound features a large proportion of long-lasting associations, Behavioral Ecology and Sociobiology 54, 396-405 (2003).

[29] D. Lusseau, The emergent properties of a dolphin social network,Proc. R. Soc. London B (suppl.) 270, S186-S188 (2003).

[30] D. Lusseau, Evidence for social role in a dolphin social network,Preprint q-bio/0607048 (http://arxiv.org/abs/qbio.PE/0607048).

[31] M. Girvan and M. E. J. Newman,Community structure in social and biological networks,Proc. Natl. Acad. Sci. USA 99, 7821-7826 (2002).

[32] M. E. J. Newman, SIAM Review 45, 167-256 (2003) and S. Boccaletti et al., Physics Reports 424, 175-308 (2006),Lada A. Adamic and Natalie Glance, "The political blogosphere and the 2004 US Election", in Proceedings of the WWW-2005 Workshop on the Weblogging Ecosystem (2005).

[33] Identifying and evaluating community structure in complex networks Karsten Steinhaeuser, Nitesh V. Chawla *University of Notre Dame, Department of Computer Science and Engineering, Interdisciplinary Center for Network Science and Applications (iCeNSA), Notre Dame, IN 46556, USAvan Dongen, S., 2000.
Graph Clustering by Flow Simulation, Ph.D. Thesis, University of Utrecht, Netherlands.

[34] Pons, P., Latapy, M., 2006. Computing communities in large networks using random walks. J. Graph Algorithms Appl. 10 (2), 191-218.

[35] M.E.J. Newman, The structure and function of complex networks".

[36] Zenklusen Rico,Complex networks:An overview.

[37] Benjamin Lam,Data mining with clustering and classification,springer,2007.

[38] Santo Fortunato, Community detection in graphs, Physics Reports 486 (2010) 75-174.

[39] Xu Liu a, Jeffrey Yi-Lin Forrest a,b, Qiang Luoc, DongYun Yi a,,* Detecting community structure using biased random merging.

[40] M.E.J Newman,The structure and function of complex networks, arXiv:cond-mat/0303516v1 [cond-mat.statmech] 25 Mar 2003.

[41] Milligan, G., Cooper, M., 1986. A study of the comparability of external criteria for hierarchical cluster analysis. Multivar. Behav. Res. 21 (4),441-458.

[42] Merrian Webster,Online Dictionary,2008.Cluster Analysis. http://www.merriam-webster-online.com

[43] Jain,A.K.,2009.Data Clustering :50 years beyond kmeans , <http://dataclustering.cse.msu.edu/papers/jainDataCluste ringPRL09.pdf>.

[44] Steinhaus,H.,1956.Sur la division des corp materials en parties.Bull.Acad.Polon.Sci.,C1,III IV,801-804.

[45] Berkhin,P.,2009. Survey of clustering data mining techniques,http://www.ee.ucr.edu/barth/EE242/.

[46] Code of the BGLL is used from the link http://findcommunities.googlepages.com. 